\title{
On the lattice structure of quantum logic
}

\author{
P. D. Finch
}

\begin{abstract}
A weak logical structure is defined as a set of boolean propositional logics in which one can define common operations of negation and implication. The set union of the boolean components of a weak logical structure is a logic of propositions which is an orthocomplemented poset, where orthocomplementation is interpreted as negation and the partial order as implication. It is shown that if one can define on this logic an operation of logical conjunction which has certain plausible properties, then the logic has the structure of an orthomodular lattice. Conversely, if the logic is an orthomodular lattice then the conjunction operation may be defined on it.
\end{abstract}

\section{Introduction}

The axiomatic development of non-relativistic quantum mechanics leads to a quantum logic which has the structure of an orthomodular poset. Such a structure can be derived from physical considerations in a number of ways, for example, as in Gunson [7], Mackey [11], Piron [12], Varadarajan [13] and Zierler [14]. Mackey [11] has given heuristic arguments indicating that this quantum logic is, in fact, not just a poset but a lattice and that, in particular, it is isomorphic to the lattice of closed subspaces of a separable infinite dimensional Hilbert space. If one assumes that the quantum logic does have the structure of a lattice, and not just that of a poset, it is not difficult to ascertain what sort of further assumptions lead to a "coordinatisation" of the logic as the lattice of closed subspaces of Hilbert space, details will be found in Jauch [8], Piron [12], Varadarajan [13] and Zierler [14]. 
There is however a need to justify the assumption of a lattice structure at a phenomenological and interpretative level. Foul is [4] discovered the close relationship between orthomodular lattices and Baer *-semigroups and in [5] showed that an involution poset is an orthomodular lattice if and only if its associated involution semigroup is a Baer *-semigroup. This important result singles out the orthomodular lattices within the class of involution posets, however it does so by a mathematical specification which is not directly interpretable as a phenomenological specification which is both meaningful and compelling within the framework of an exiomatic development of quantum mechanics.

In this paper we relate the lattice structure of quantum logic to the existence of an operation of logical conjunction between its propositions. We do this in the following way. In Finch [2] it is shown how orthomodular posets arise naturally in the study of certain sets of boolean logics in which one can define common operations of implication and negation. In this paper we show that if, in addition, one wants to define an operation of "logical confunction" with certain plausible properties then one obtains a full Sasaki set of projections in the sense of Finch [3]; this fact leads directly to a logic which has the structure of an orthomodular lattice. To obtain this result one needs only to start with an orthocomplemented poset rather than an orthomodular poset, and so in deriving it we are able to weaken the concept of a logical structure in Finch [2] which was designed to lead to a logic having the structure of poset which was not just orthocomplemented but also orthomodular.

\section{Weak logical structures}

By a boolean logic $L$ we mean a boolean algebra of propositions in which the boolean lattice operations of join, meet and orthocomplementation correspond to the logical operations of disjunction, conjunction and negation respectively. As is well known the ordering in $L$ may be interpreted as a logical relation of implication between the propositions of $L$. We use $I$ and 0 to denote the greatest and least elements of $L$.

In what follows we consider an indexed set $L=\left\{L_{\gamma}: \gamma \in \Gamma\right\}$ of boolean logics $L_{\gamma}$, which are not necessarily disjoint, such that one can 
define common operations of negation and implication in $U\left\{L_{\gamma}: \gamma \in \Gamma\right\}$. In order to specify the structure of $L$ we need to refer to the logical operations in each $L_{\gamma}$; to avoid ambiguity we denote order in $L_{\gamma}$ by $\omega_{\gamma}$ and the orthocomplementation in $L_{\gamma}$ by $N_{\gamma}$. Thus for $x$ and $y$ in $L_{\gamma}$ we mean by "xw $y$ " that in the logic $L_{\gamma}$ the proposition $x$ implies the proposition $y$ and, to avoid unnecessary repetition, we adopt the convention that whenever an expression such as " $x \omega{ }^{2} y$ " occurs it is to be understood that $x$ and $y$ are both in $L_{\gamma}$. Similarly for $x$ in $L_{\gamma}$ " $N_{\gamma} x "$ denotes the negation in the logic $L_{\gamma}$ of the proposition $x$, it being understood that $x$ is in the logic $L_{\gamma}$.

DEFINITION. A weak logical structure is an indexed set $L=\left\{L_{\gamma}: \gamma \in \Gamma\right\}$ of boolean logics with the following properties,

(i) each $L_{\gamma}$ has the same least element 0 ,

(ii) if $x$ and $y$ belong to $L_{\alpha} \cap L_{\beta}$ then $x \omega_{\alpha} y$ if and only if $x \omega_{\beta} y$

(iii) if $x \omega_{\alpha} y$ and $y \omega_{\beta} z$ there is $\gamma$ in $\Gamma$ such that $x \omega_{\gamma} z$, (iv) if $x$ belongs to $L_{\alpha} \cap L_{\beta}$ then $N_{\alpha} x=N_{\beta} x$.

Note that the conditions for a weak logical structure form a proper subset of those for a logical structure in Finch [2].

Let $L$ be a weak logical structure and write

$$
L=U\left\{L_{\gamma}: \gamma \in \Gamma\right\},
$$

we call $L$ the logic associated with the weak logical structure $L$. One can introduce a partial ordering into $L$ by decreeing that for $x, y$ in $L$ one has $x \leqq y$ if and only if there is a $\gamma$ in $\Gamma$ such that $x \omega y$. Because of (i) the common 0 of the $L_{V}$ is the least element of $L$. By (iv) one can define a map $N: L \rightarrow L$ by asserting that for each $x$ in $L$ one has $N x=N_{\gamma} x$ for any $L_{\gamma}$ which contains $x$. In particular the 
common $I=N O$ of the $L_{\gamma}$ is the greatest element of $L$. The map $N$ has the following properties

(a) $N N x=x$ for each $x$ in $L$,

(b) $x \leqq y$ implies $N y \leqq N x$,

(c) 1 is the 1.u.b. in $L$ of $x$ and $N x$.

Of these properties (a) and (b) are obvious consequences of the corresponding facts about each $N_{\gamma}$ in its parent logic $L_{\gamma}$. To prove (c) let $y$ be any upper bound in $L$ to $x$ and $N x$. There are $\alpha$ and $B$ in $\Gamma$ such that $x \omega_{\alpha} y$ and $(N x) \omega_{\beta} y$. But $(N x) \omega_{\beta} y$ implies $(N y) \omega_{\beta} x$ and then, by (iii),

$$
\text { (Ny) } \omega_{\beta} x \quad \& \quad x \omega_{\alpha} y \Rightarrow \exists_{\gamma} \cdot(N y) \omega_{\gamma} y \text {. }
$$

Since $N y=N_{\gamma} y$ and $L_{\gamma}$ is a boolean algebra we have $N_{\gamma} y=0$, that is $y=1$ and this is the desired result.

In the terminology of lattice theory the three properties $(a),(b)$ and (c) mean that the map $N$ is an orthocomplementation of the poset $L$. When $x \leqq y$ we say that $x$ implies $y$ in the logic $L$ and for each $x$ in $L$ call $N x$ the negation in $L$ of the proposition $x$. In accordance with the usual terminology, Birkhoff [1], we sometimes write $x^{\perp}$ instead of $N x$ and $x \perp y$ to mean $x \leqq y^{\perp}$.

\section{Logical conjunction}

Let $L$ be the logic associated with a weak logical structure; we investigate the consequences of the introduction into $L$ of a binary operation " $x \circ y$ " of "logical confunction" which has the properties

(1) $1 \circ x=x$ for all $x$ in $L$,

(2) $x \leqq y$ implies $x \circ z \leqq y \circ z$ for all $z$ in $L$,

(3) $x \leqq y$ implies $(z \circ y) \circ x=z \circ x$ for all $z$ in $L$,

(4) $\{N(x \circ y)\} \circ y \leqq N x$.

We remark that although we read " $x \circ y$ " as $" x$ and $y$ ", we do not identify $" x \circ y$ " and $" y \circ x$ " nor do we assume that logical conjunction 
is associative. Indeed it will turn out that $x \circ y=y \circ x$ if and only if there is a boolean subalgebra of $L$ which contains $x$ and $y$; in that case $x \circ y=x \wedge y$. The four properties listed above are readily translated into words, each of them then has an obvious plausibility in the sense that it is difficult to envisage a "useful" operation of logical conjunction which did not have these properties. However our main purpose is not to argue the plausibility of interpreting our operation as one of logical conjunction, but to study the effect of its existence on the structure of the logic $L$. With this end in view we establish the following

THEOREM. Let $L$ be the logic associated with a weak logical structure. Then $L$ is an orthomodular lattice if (and only if) it admits an operation of logical conjunction with the four properties listed above.

Proof. Assume that $L$ admits an operation of logical conjunction with the stated properties. For each $x$ in $L$ define a map $\pi_{x}: L \rightarrow L$ by writing $y \pi_{x}=y \circ x$ for each $y$ in $L$. One now verifies easily that $S=\left\{\pi_{x}: x \in L\right\}$ is a full Sasaki set of projections on $L$ in the sense of Finch [3]. It follows at once, from the theorem of that paper, that $L$ is an orthomodular lattice and that

$$
y \circ x=(y \vee N x) \wedge x
$$

Conversely if $L$ is an orthomodular lattice this equation defines an operation of logical conjunction with the four properties listed above.

REMARK. Note that if $L$ is an orthomodular lattice and $x, y$ in $L$ are simultaneously verifiable in the sense of Varadarajan [13], that is there exists a boolean subalgebra of $L$ which contains $x$ and $y$ then

$$
x \circ y=x \wedge y=y \circ x \text {. }
$$

Conversely if $x \circ y=y \circ x$ then

$$
x \circ y=(x \circ y) \wedge y=(y \circ x) \wedge y=x \wedge y
$$

and then

$$
y \wedge(x \wedge y)^{\perp}=y \wedge\left\{\left(x^{\perp} \wedge y\right) \vee y^{\perp}\right\}=x^{\perp} \wedge y \leqq x^{\perp}
$$

It follows from Lemma (6.7) of Varadarajan [13] that $x$ and $y$ are simultaneously verifiable. In particular therefore, $x \circ y=y \circ x$ for all 
$x, y$ in $L$ if and only if $L$ is a boolean logic. It is possible to define simultaneous verifiability (or equivalently "compatibility") by the lattice equivalent of the equation $x \circ y=y \circ x$; this is done, for example, by Jauch and Piron [9]. However, those authors interpret the lattice meet operation as the conjunction in their quantum logic.

\section{Concluding remarks}

Let $L$ be the logic associated with a weak logical structure and suppose that $L$ admits the operation of logical conjunction defined above. It is then possible to introduce a binary operation $x * y$ of "logical disjunction" between the elements of $L$ by the equation

$$
x * y=\left(x^{\perp} \circ y^{\perp}\right)^{\perp} \text {. }
$$

Note that

$$
x^{\perp} * y^{\perp}=1 \Leftrightarrow x \circ y=0 \Leftrightarrow x \leqq y .
$$

This suggests that one could base the laws for quantum logic on either of the operations ' $*$ ' and ' 0 '. According to Gericke [6] a description of quantum logic based on the ' $*$ ' operation was carried out in Kunsemüller [10]. However, from Gericke's account of this work it would appear that Kunsemüller interpreted the ' $*$ ' operation as one of logical subjunction since it generalised the usual subjunction, $x^{\perp} \vee y$, in a boolean logic.

In quantum mechanics probability is introduced as a non-negative function $\mu$ on an orthomodular lattice such that $\mu(1)=1$ and

$$
\mu(x \vee y)=\mu(x)+\mu(x)
$$

whenever $x \perp y$, that is $x \leqq y^{\perp}$. In fact one usually requires o-additivity over countably many orthogonal joins, but for the purpose of these remarks finite additivity will suffice. The difference between probabilities in this context and that of a boolean logic comes about because one coes not have the familiar equation

$$
\mu(x \vee y)+\mu(x \wedge y)=\mu(x)+\mu(y),
$$

which holds in boolean logics and for valuations on lattices. We show now how the use of the ' $O$ ' and ' $*$ ' operations permits a formal connection between the theory of valuations on lattices and probabilities on 
orthomodular lattice.

By on ortho-valuation on an orthomodular lattice $L$ we will mean a real-valued function $f$ on $L$ such that

$$
f(x * y)+f(y \circ x)=f(x)+f(y) .
$$

We prove the following lemma which is a formal analogue of a well-known result for valuations on relatively complemented lattices, see, for example, Birkhoff [1].

LEMMA. A real-valued function $f$ on an orthomodular lattice is an ortho-valuation if (and only if)

$$
f(x \vee y)=f(x)+f(y)
$$

whenever $x \perp y$.

$$
\begin{gathered}
\text { Proof. } x * y=y \vee\left(y^{\perp} \wedge x\right) \text { and } y \perp\left(y^{\perp} \wedge x\right) \text {, thus } \\
f(x * y)=f(y)+f\left(y^{\perp} \wedge x\right) .
\end{gathered}
$$

Again

$$
x=(y \circ x) \vee\left(y^{\perp} \wedge x\right)
$$

and $(y \circ x) \perp\left(y^{\perp} \wedge x\right)$, thus

$$
f(y \circ x)=f(x)-f\left(y^{\perp} \wedge x\right) .
$$

This establishes that $f$ is an ortho-valuation.

This result and the formal similarity between the definitions of an ortho-valuation and a valuation suggest some deeper connection. In fact one can establish in orthomodular lattices some curious analogues of well-known results in modular lattices by replacing lattice meet and join by the 'o' and ' *' operations respectively. Details of some of these will be given in a later publication.

\section{References}

[1] Garrett Birkhoff, Lattice theory, (Amer. Math. Soc. Colloquium Publications, Vol. 25 3rd edition, 1967). 
[2] P.D. Finch, "On the structure of quantum logic", J. Symbolic Logic 34 (1969), 275-282.

[3] P.D. Finch, "Sasaki projections on orthocomplemented posets", BuZZ. Austral. Math. Soc. 1 (1969), 319-324.

[4] David J. Foulis, "Baer *-semigroups", Proc. Amer. Math. Soc. 11 $(1960), 648-654$.

[5] David J. Foulis, "A note on orthomodular lattices", Portugal. Math. 21 (1962), 65-72.

[6] Helmuth Gericke, Lattice theory (Harrap, London, 1966).

[7] J. Gunson, "On the algebraic structure of quantum mechanics", Comm. Math. Phys. 6 (1967), 262-285.

[8] Joseph M. Jauch, Foundations of quantrom mechanics (Addison-Wesley, Reading, Massachusetts, 1968).

[9] J.M. Jauch and C. Piron, "Can hidden variables be excluded in quantum mechanics?", HeZv. Phys. Acta. 36 (1963), 827-837.

[10] H. Kunsemüller, "Zur logischen Deutung der Quantenmechanik", (Diss. Phil. Univ. Hamburg, 1959).

[11] George W. Mackey, Mathematical foundations of quantum mechanics (Benjamin, New York, 1963).

[12] C. Piron, "Axiomatique quantique", Helv. Phys. Acta. 37 (1964), 439-468.

[13] V.S. Varadarajan, Geometry of quantion theory (Van Nostrand, Princeton, New Jersey, 1968).

[14] Neal Zierler, "Axioms for non-relativistic quantum mechanics", Pacific J. Math. 2 (1961), 1151-1169.

Monash University,

clayton, victoria. 